\title{
Higher-Order Squeezing of a Generic Quadratically-Coupled Optomechanical System
}

\author{
Kousik Mukherjee ${ }^{1,2}$, Paresh Chandra Jana ${ }^{1}$ \\ Department of Physics and Technophysics, Vidyasagar University, Midnapore, 721102, India ${ }^{l}$ \\ Department of Physics, Govt. General Degree College Gopiballavpur-II, 721517, India
}

\begin{abstract}
Using short-time dynamics and analytical solution of Heisenberg equation of motion for the Hamiltonian of quadratically-coupled optomechanical system for different field modes, we have investigated the existence of higher-order single mode squeezing, sum squeezing and difference squeezing in absence of driving and dissipation. Depth of squeezing increases with order number for higher-order single mode squeezing. Squeezing factor exhibits a series of revival-collapse phenomena for single mode, which becomes more pronounced as order number increases. In case of sum squeezing amounts of squeezing is greater than single mode higher-order squeezing $(n=2)$. It is also greater than from difference squeezing for same set of interaction parameters. Sum squeezing is prominently better for extracting information regarding squeezing.
\end{abstract}

Keywords: nonclassical, higher-order squeezing, optomechanical.

\section{Introduction}

A single mode light has two components in terms of two quadrature operators which obey Heisenberg uncertainty relation and are non-commutating [1,2]. The variance of quadrature has equal lower bound, for nonclassical character of the optical field. Variance of one quadrature may be less than the lower bound due to cost of other variance. This nonclassical feature is named as squeezing. Nonclassical fields are those for which Glauber-Sudarshan P function is negative or more singular than delta function. As P function is not observed directly through experiments, it is necessary to talk about other criteria to characterize nonclassicality. These are Fano Factor, Wigner function negativity etc. There are several nonclassical features such as entanglement, antibunching, quantum beats, collapse-revivals [3] etc. Revivals are true quantum effects, which are due to discreteness of particle number states. It is well known that uncertainty of any one of the quadrature in the squeezed state is reduced in comparison to the coherent state. Any one of the squeezed state be less noisy than coherent one. Squeezing may be of different nature such as amplitude squeezing, phase squeezing, spin squeezing, sum squeezing, difference squeezing etc. These nonclassical features have potential application in interferometric technique [4], optical waveguide tap [5], optical communication [6], quantum cryptography [7], quantum dense coding [8], teleport entangled quantum bits [9], nonlinear beam splitter [10], multi-wave mixing Process [11], enhance sensitivity of the gravitational wave detector $[12,13]$. Squeezing has been theoretically predicted and experimentally observed in different nonlinear optical processes such as four-wave mixing [14], free electron laser[15], Optomechanical system[16, 17] etc. Optomechanical system (OMS) lies at the boundary between nanoscience and quantum optics. It also provides quantum effects at macroscopic scale. In OMS there are coherent coupling between electromagnetic and mechanical degrees of freedom via radiation pressure $[18,19]$. In general, optomechanical coupling may be linear or quadratic according to the coupling term proportional to $x$ or $x^{2}$ where $x$ is the mechanical displacement of the membrane. For quadratic coupling there will be a change in resonant frequency of the cavity resonator. Recently different methods or proposals are stated to increase the coupling strength for studying the features of the optomechanics in single photon strong coupling regime [20,21]. As there is photon phonon interaction in the cavity, there is also a possibility of phonon squeezing. It is observed that phonon squeezed states reduces quantum noise in solids [22]. Lower order squeezing in quadratic-coupled OMS for different field modes have already been studied [16]. In this article we focus our attention on higher-order squeezing. For higher-order the definition of amplitude-squared squeezing, amplitude-cube squeezing, $n$th order single mode squeezing [23] are well known. Higher-order two-mode squeezing has been studied in-terms of sum and difference squeezing [24]. Regarding the generation of higher-order squeezed states for electromagnetic field, a lot of work has been done on both experimental and theoretical investigations. Hillery has shown that amplitude squared squeezed states can be used for reduction of noise for nonlinear optical systems [25]. Higher-order squeezing has useful application in prototype gravitational wave detection [26], higher harmonic generation [27, 28], hyper- Raman scattering [29], parametric amplification [30], Six-wave mixing process [31], and so forth.The present article is organized as follows: at first we describe the model Hamiltonian of quadratically coupled OMS and its perturbative solutions for Heisenberg equation of motion corresponds to various field modes. Using the criteria for different higher order squeezing we have investigated the existence of squeezed states for various field modes. At last, we have discussed results with conclusions. 


\section{The Model and its solutions}

Quadratic-coupled OMS is a system (a cavity) in which a dielectric membrane (may be $\mathrm{Si}_{3} N_{4}$ [32]) is placed at the middle between two macroscopic, rigid and high-finesse mirrors i.e. a Fabry-P'erot cavity. The coupling between mechanical motion of the membrane and the optical cavity depends on the position of the membrane, relative to a node (or antinode) of the intra-cavity standing wave. The Hamiltonian for such a system is given by $(\hbar=1)[32,33]$

$H=\omega_{c} a^{\dagger} a+\omega_{m} b^{\dagger} b+g a^{\dagger} a\left(b^{\dagger}+b\right)^{2}+i E\left(a^{\dagger} e^{-i \omega_{d} t}-a e^{i \omega_{d} t}\right)$

The single mode cavity field is characterized by the annihilation (creation) operators $a\left(a^{\dagger}\right)$ and the field operators $b\left(b^{\dagger}\right)$ for the mechanical motion of the membrane having resonant frequency $\omega_{c}$ and that of mechanical mode $\omega_{m}$ respectively. These operators are satisfying bosonic commutation relation $\left[a, a^{\dagger}\right]=$ $\left[b, b^{\dagger}\right]=1 . g$ is the coupling strength between cavity field and the membrane. Recent studies [34, 35] shown that the value of $g$ can be order of several $\mathrm{kHz}$. For near field optomechanical system there is a proposal to estimate the coupling strength to be of the order of few $\mathrm{MHz}$ [21]. E is the external driving parameter which drives the system by frequency $\omega_{d}$. As optomechanical system is closed, the environmental effects are negligible and experiment [36] is directed towards optomechanical system in which environmental effects are less. Here we consider system Hamiltonian in absence of zero driving as used in references [16, 37, 38, 39] in different situation for optomechanical system. So, in absence of driving (i.e. $E=0$ ) and other losses, the Hamiltonian of the system takes the form

$$
H=\omega_{c} a^{\dagger} a+\omega_{m} b^{\dagger} b+g a^{\dagger} a\left(b^{\dagger}+b\right)^{2}
$$

In order to investigate the existence of higher-order squeezing of a generic quadratically coupled OMS we use Heisenberg equations of motion for cavity field mode and mechanical mode which corresponds to the above Hamiltonian. We write the equations of motion for $a$ and $b$ as follows:

$$
\begin{gathered}
\dot{a}(t)=-i\left[\omega_{c} a(t)+g a(t)\left\{b^{\dagger 2}(t)+b^{2}(t)+2 b^{\dagger}(t) b(t)+1\right\}\right] \\
\dot{b}(t)=-i\left[\omega_{m} b(t)+2 g a^{\dagger}(t) a(t)\left\{b^{\dagger}(t)+b(t)\right\}\right]
\end{gathered}
$$

Using short-time dynamics we may expand any operator $a(t)$ in Taylor series as $a(t)=a(0)+i t[H, a(0)]+$ $\cdots$. From the evaluation of commutation we get the time development of operator $a(t)$ in-terms of $a(0)$.

The solutions of the equation (3) assumes the following form

$$
\begin{aligned}
& a(t)=g_{1} a(0)+g_{2} a(0) b^{\dagger 2}(0)+g_{3} a(0) b^{2}(0)+g_{4} a(0) b^{\dagger}(0) b(0)+g_{5} a(0) \\
& b(t)=l_{1} b(0)+l_{2} a^{\dagger}(0) a(0) b(0)+l_{2} a^{\dagger}(0) a(0) b^{\dagger}(0)
\end{aligned}
$$

where the parameters $g_{i}(i=1, \ldots, 5)$ and $l_{i}(i=1, \ldots, 3)$ are function of time and can be evaluated from initial boundary conditions $g_{1}=l_{1}=1$ and $g_{i}=l_{i}=(i=2, \ldots, 5)$.

The solutions of $g_{i}(t)$ and $l_{i}(t)$ are given by

$g_{1}(t)=e^{-i \omega_{c} t} ; g_{2}(t)=\frac{g}{2 \omega_{m}} g_{1}(t) G(t) ; g_{3}(t)=-\frac{g}{2 \omega_{m}} g_{1}(t) G^{*}(t) ; g_{4}(t)=-2 i g t g_{1}(t)$;

$g_{5}(t)=-i g t g_{1}(t) ; l_{1}(t)=e^{-i \omega_{m} t} ; l_{2}(t)=-2 i g t l_{1}(t) ; l_{3}(t)=\frac{g}{\omega_{m}}\left[l_{1}(t)-l_{1}^{*}(t)\right]$

where $G(t)=1-e^{2 i \omega_{m} t}$

The above solutions are valid up to first order in $g$ such that $g t<1$ and perturbation calculation holds good. For checking the solutions we use Equal time commutation relation (ETCR) as $\left[a(t), a^{\dagger}(t)\right]=\left[b(t), b^{\dagger}(t)\right]=$ 1 has been used. Here the operator $a(0)$ commutes with $b^{\dagger}(0)$ and $b(0)$. Similarly, $b(0)$ commutes with $a^{\dagger}(0)$ and $a(0)$.

During the analytical solution, we choose parameters of the system based on experiment for optomechanical systems, which are used in references $[20,32,40]$.

\section{Quadrature squeezing}

Squeezing is defined as a quantum mechanical fluctuation of quadratures. For investigating squeezing we have to define two quadratures $X_{1}$ and $X_{2}$, which express the real(electric) and imaginary(magnetic) parts of the radiation field respectively. We assume the quadrature operators satisfy the following commutation relation $\left[X_{1}, X_{2}\right]=\frac{1}{2} C$, where $\mathrm{C}$ is an operator or $\mathrm{C}$-number. Using the uncertainty relation the commutation rule can be written as

$$
\left\langle\left(\Delta X_{1}\right)^{2}\right\rangle\left\langle\left(\Delta X_{2}\right)^{2}\right\rangle \geq \frac{1}{16}|\langle C\rangle|^{2}
$$

where $\left\langle\left(\Delta X_{i}\right)^{2}\right\rangle=\left\langle X_{i}{ }^{2}\right\rangle-\left\langle X_{i}\right\rangle^{2}$. The system is said to be squeezed in the quadrature if

$$
\left\langle\left(\Delta X_{i}\right)^{2}\right\rangle-\frac{1}{4}|\langle C\rangle| \leq 0
$$

where equality sign holds for minimum uncertainty. 


\section{III.1 The n-th order single mode squeezing:}

For $\mathrm{n}$-th order single mode squeezing the operators are [23]

$$
\begin{gathered}
X_{1}=\frac{1}{2}\left\{a^{n}(t)+a^{\dagger n}(t)\right\}, X_{2}=\frac{1}{2 i}\left\{a^{n}(t)-a^{\dagger n}(t)\right\} \\
C=a^{n}(t) a^{\dagger n}(t)-a^{\dagger n}(t) a^{n}(t)
\end{gathered}
$$

The field mode is said to be squeezed in the $X_{i}$ direction if $S_{i}(n)<0$ where we define $S_{i}(n)=\left\langle\left(\Delta X_{i}\right)^{2}\right\rangle-$ $\frac{1}{4}|\langle C\rangle| \leq 0 \quad$ with $i=1,2$.

For $n=2$ i.e. for amplitude squared squeezing the above condition takes the form

$$
\left\langle\left(\Delta X_{i a}\right)^{2}\right\rangle-\left\langle\left(N_{a}+\frac{1}{2}\right)\right\rangle<0
$$

Where $i=1,2$. Similarly, for $n=3$ i.e. for the state to be amplitude cube squeezed if

$$
\left\langle\left(\Delta X_{i a}(t)\right)^{2}\right\rangle-\left\langle\left(9 N_{a}^{2}+9 N_{a}+6\right)\right\rangle<0
$$

The number operator for the field mode $a$ and $b$ are

$$
\begin{gathered}
N_{a}=a^{\dagger}(t) a(t)=a^{\dagger}(0) a(0) g_{1}^{*} g_{1}+\left[a^{\dagger}(0) a(0) b^{2}(0) g_{2}^{*} g_{1}+a^{\dagger}(0) a(0) b^{\dagger 2}(0) g_{3}^{*} g_{1}\right. \\
\left.+a^{\dagger}(0) a(0) b^{\dagger}(0) b(0) g_{4}^{*} g_{1}+a^{\dagger}(0) a(0) g_{5}^{*} g_{1}+h . c .\right] \\
N_{b}=b^{\dagger}(t) b(t)=b^{\dagger}(0) b(0) l_{1}^{*} l_{1}+\left[a^{\dagger}(0) a(0) b^{\dagger}(0) b(0) l_{2}^{*} l_{1}+a^{\dagger}(0) a(0) b^{2}(0) l_{3}^{*} l_{1}+h . c\right]
\end{gathered}
$$

In order to calculate the fluctuations of the quadratures, we assume that photon and phonon modes are initially in coherent state (i.e. composite bosonic field consisting photon and phonon). So initial state is the product of two coherent states $|\alpha\rangle \otimes|\beta\rangle$ where $|\alpha\rangle$ and $|\beta\rangle$ are the eigenkets of field operators $a$ and $b$ respectively. If the operator $a(t)$ operates on the state, it gives complex eigenvalue $\alpha$. Using these we obtain

$$
\begin{gathered}
\left(\begin{array}{c}
s_{1 a}(n) \\
s_{2 a}(n)
\end{array}\right)=0 \\
\left(\begin{array}{c}
s_{1 b}(n) \\
s_{2 b}(n)
\end{array}\right)= \pm \frac{n^{2}}{4}|\alpha|^{2}\left(l_{3} l_{1}^{2 n-1} \beta^{2 n-2}+\text { c.c. }\right)
\end{gathered}
$$

where c.c. indicates complex conjugates.

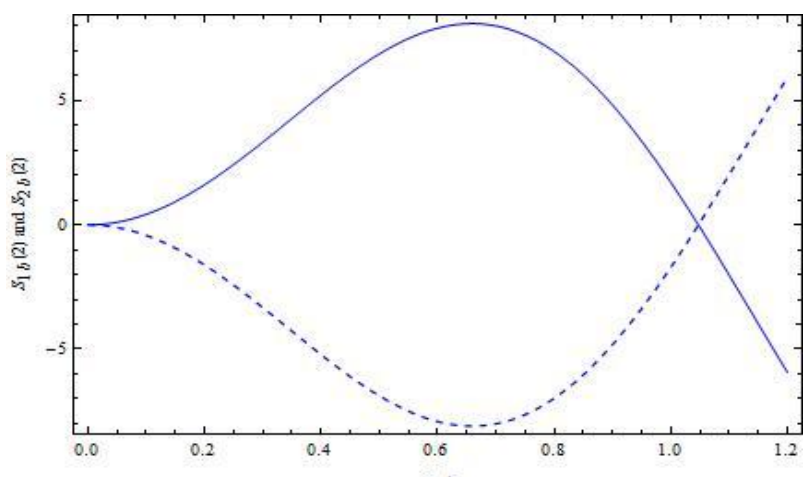

Figure 1: Plot of squeezing parameter $S_{1 b}(2)$ and $S_{2 b}(2)$ with $\omega_{m} t$ for $b$ mode with $\mathrm{n}=2, \alpha=3, \beta=2$,

$$
g=2 \pi \times 10 \mathrm{KHz} \text { and } \frac{g}{\omega_{m}}=0.1 \text {. }
$$

From above results it is evident that there is no signature of higher-order single mode squeezing for cavity field mode. To investigate the possibility of higher-order single mode squeezing for $b$ mode we have plotted the result of the equation (13) in figures - 1,2 as a function of $\omega_{m} t$. Figure 1 and 2 represent the variation of squeezing parameters for $n=2$ i.e. amplitude squared squeezing and $n=3$ i.e. amplitude cube squeezing respectively. From these variations one can observe that any quadrature of the mechanical mode always be squeezed due to cost of other which is a consequence of Heisenberg uncertainty relation. The depth of squeezing increases with $\beta$, order number $n$ and also with coupling strength $g$. From figures 1 and 2 it is clear that amount of squeezing increases almost ten times when we increase order number from $n=2$ to $n=3$ for same values of interaction parameters. If we consider $\beta$ as real it can be said that the term $n^{2} \beta^{2 n-2}$ plays the role of amplification factor. Again, for a given order number $n$ the coupling strength $g$ plays the same role. The presence of the function $l_{3}(t)$ in the expression is the key factor for that. It is also seen that as order number $n$ increases, time period for oscillations decreases due to decrease in energy exchange. Here monotonous increasing of envelope for both the quadratures is observed due to truncation of perturbation calculation for coupling strength $g$ in the solutions. Figure 3 represents the variation of the equation (13) as a function of time. From this representation it is observed that there is a collapse-revival phenomenon. The occurrence of this 
phenomenon in quadratically coupled OMS may be explained as follows. Here $S_{i b}(n)$ is function of time which includes two forms of periodic function which are $f\left(\omega_{m} t\right)$ and $f\left(n \omega_{m} t\right)$. For a fixed value of membrane frequency $\omega_{m}$, when the period of energy exchange between the modes decreases many oscillations occur, up to interaction time $\omega_{m} t=s \pi$ ( is positive integer). At this moment the field is trapped due to presence of the nonlinearity in the cavity membrane, so $S_{i b}(n)$ collapse. As phononic interaction proceeds, this phenomenon repeats periodically. The number of revival pattern increases with increasing the order number $n$, because the period of each revival is controlled by the factor $t=\frac{2 \pi}{(2 n-1) \omega_{m}}$. According to our calculation the factor $l_{3}(t)$ plays an important role for revival phenomenon and envelope function $l_{1}(t)$ provides main contribution to the collapse.

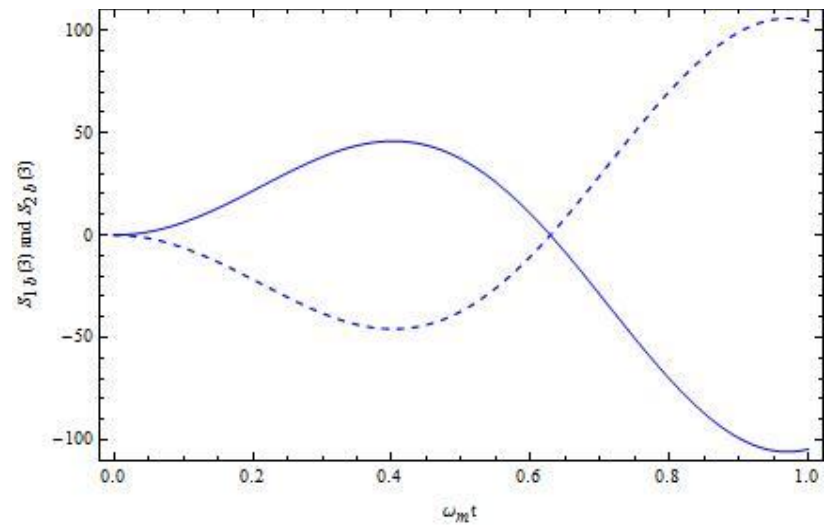

Figure 2: Plot of squeezing parameter $S_{1 b}(3)$ and $S_{2 b}(3)$ with $\omega_{m} t$ for $b$ mode with $\mathrm{n}=3, \alpha=3, \beta=2$, $g=2 \pi \times 10 \mathrm{KHz}$ and $\frac{g}{\omega_{m}}=0.1$

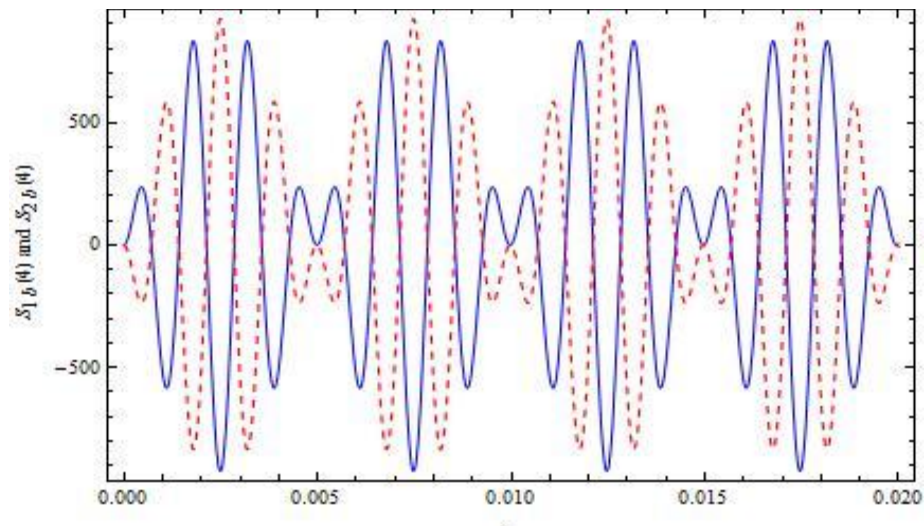

Figure 2: Plot of squeezing parameter $S_{1 b}(4)$ and $S_{2 b}(4)$ with $t(\mathrm{~ms})$ for $b$ mode with $\mathrm{n}=4, \alpha=3, \beta=2$, $g=2 \pi \times 10 \mathrm{KHz}$ and $\frac{g}{\omega_{m}}=0.1$.

\section{III.2 Sum squeezing and Difference squeezing:}

In this portion we have to find out existence of sum squeezing and difference squeezing of a generic quadratically-coupled OMS and how its depth depends on coupling strength $g$. Here we consider cavity field mode is initially coherent and mechanical mode is in vacuum state. So, initial state is $|\alpha\rangle \otimes|0\rangle$ where eigenket of field operator $a$ is $|\alpha\rangle$.

For sum-squeezing the quadrature operators $Y_{i}$ and $C$ have the form [24]

$$
\begin{gathered}
Y_{1}=\frac{1}{2}\left\{a(t) b(t)+a^{\dagger}(t) b^{\dagger}(t)\right\}, Y_{2}=\frac{1}{2 i}\left\{a(t) b(t)-a^{\dagger}(t) b^{\dagger}(t)\right\}, \\
C=N_{a}+N_{b}+1
\end{gathered}
$$

The system is said to be sum squeezed in the $Y_{i}$ direction if $S_{i_{a b}}<0$ where we define squeezing factor $S_{i_{a b}}=$ $\left\langle\left(\Delta Y_{i}\right)^{2}\right\rangle-\frac{1}{4}|\langle C\rangle| \leq 0 \quad$ with $i=1,2$. Using above squeezing conditions and solutions we find that

$$
\left(\begin{array}{l}
s_{1_{a b}} \\
s_{2} a b
\end{array}\right)= \pm \frac{1}{4}\left[g_{1}^{2} l_{3} l_{1} \alpha^{2}\left(2+|\alpha|^{2}\right)+\text { c.c. }\right]
$$




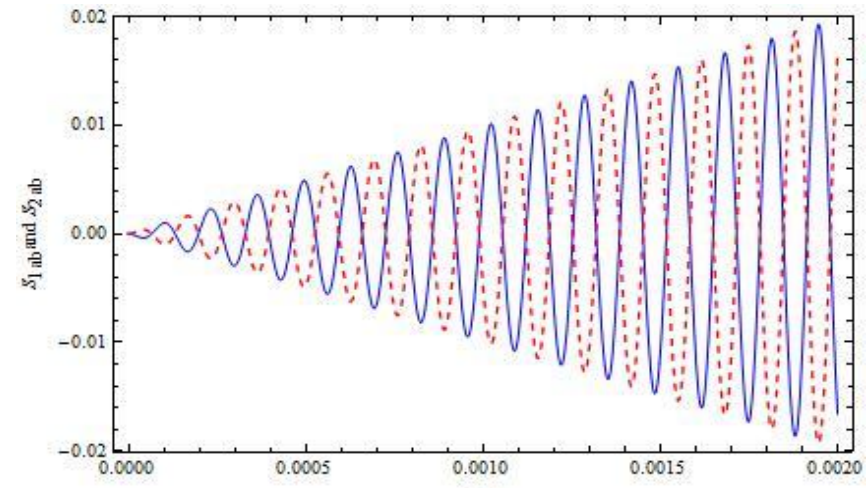

$\omega_{m}{ }^{2}$

Figure 4: Plot of squeezing parameter $S_{1_{a b}}$ and $S_{2_{a b}}$ with $\omega_{m} t$ for $a b$ compound mode with $\alpha=3, g=2 \pi \times$ $10 \mathrm{KHz}$ and $\frac{g}{\omega_{m}}=0.1$

In order to illustrate the possibility of observing sum squeezing for cavity field mode and mechanical mode we have plotted $S_{i_{a b}}$ from equation (15) as function of $\omega_{m} t$ in figure 4. From the plot it is cleared that sum squeezing is observed for the system and output of sum-squeezing depends on sum of the frequency of the cavity mode and mechanical mode i.e. $\omega_{c}+\omega_{m}$. The amount of squeezing is more pronounced with increasing of coupling strength $g$ and same explanation for monotonous increasing of envelope of the curve has already been discussed. Here, we also note that although sum squeezing is one type of higher-order squeezing but the depth of squeezing is more pronounced for sum squeezing than $n$th order single mode phonon squeezing for $n=2$ under same values of interaction parameter. Although both the processesses of interactions are instantaneous but energy transfer for photon-phonon interaction is more than phonon-phonon interaction. The amount of squeezing also depends on relative phase angle of $\alpha$.

Using quadrature operators $Z_{1}, Z_{2}$ and , we have to investigate possibilities of difference squeezed states for the quadratically coupled OMS, where the operator take the form [24] as

$Z_{1}=\frac{1}{2}\left\{a(t) b^{\dagger}(t)+a^{\dagger}(t) b(t)\right\}, \quad Z_{2}=\frac{1}{2 i}\left\{a(t) b^{\dagger}(t)-a^{\dagger}(t) b(t)\right\}$,

$$
C=N_{b}-N_{a}
$$

The system is said to be sum squeezed in the $Z_{i}$ direction if $Q_{i_{a b}}<0$ where we define squeezing factor $Q_{i_{a b}}=$ $\left\langle\left(\Delta Z_{i}\right)^{2}\right\rangle-\frac{1}{4} \quad|\langle C\rangle| \leq 0 \quad$ with $i=1,2$. Using above squeezing conditions and solutions we obtain

$$
\left(\begin{array}{c}
Q_{1} \\
Q_{a b}
\end{array}\right)= \pm \frac{1}{4}\left[g_{1}^{2} l_{1}^{*} l_{3}^{*} \alpha^{*} \alpha^{3}+\text { c.c. }\right]
$$

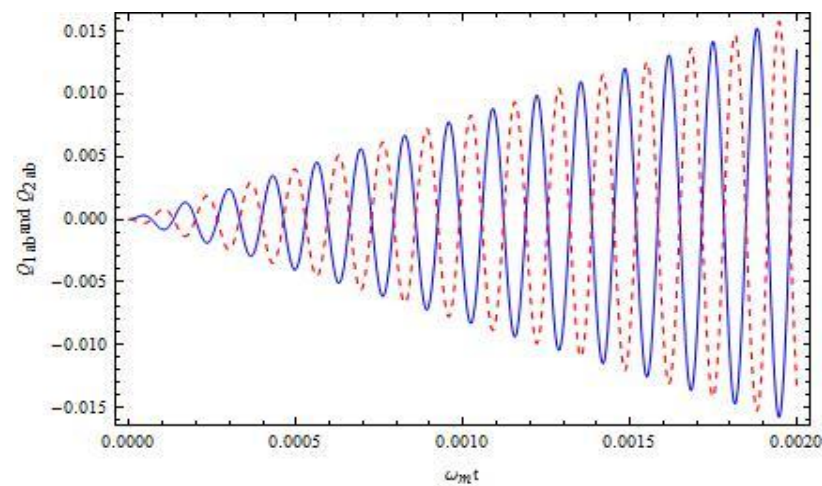

Figure 5: Plot of squeezing parameter $Q_{1_{a b}}$ and $Q_{2_{a b}}$ with $\omega_{m} t$ for $a b$ compound mode with $\alpha=3, g=2 \pi \times$ $10 \mathrm{KHz}$ and $\frac{g}{\omega_{m}}=0.1$.

To find out existence of difference squeezing in present system we have plotted the $Q_{i_{a b}}$ as given by equation (17) as a function of $\omega_{m} t$ in figure 5. From the above plot it is observed that the squeezing depends on difference of frequency of the cavity field mode and mechanical mode. In our calculation $N_{a}>N_{b}$ which satisfies the condition of difference squeezing as stated in reference [24]. For difference squeezing, depth of squeezing is more than 2nd order single mode (b-mode) but almost fifty percent of the sum squeezing for same values of interaction parameters. This is probably due to dephasing of photon and phonon. Difference squeezing also depends on relative phase of $\alpha$ i.e. if we replace $\alpha$ by $|\alpha| e^{i \pi}$. 


\section{Conclusions}

In this article, for the first time higher-order squeezing is investigated by us of a generic quadraticallycoupled OMS. For $n$th order single mode squeezing it is found that the depth of squeezing increases as order number increases and with decreasing time period of fluctuations. Due to nonlinearity of dielectric constant of the membrane squeezing factors exhibit revival-collapse phenomenon. The number of revival patterns increases with order number and the depth of it depend on both the order number and also the coupling strength. Sum squeezing provides sum-frequency and difference squeezing gives difference-frequency generation. The amount of all the nonclassical effects i.e. three types of squeezing increases considerably with coupling strength. In case of sum squeezing the depth of squeezing is more than other two types i.e. 2nd order single mode and difference squeezing for same values of interaction parameters. So, sum squeezing is the best measure for extracting information regarding squeezing for quadratically-coupled OMS.

\section{References}

[1]. V.V.Dodonov, J.Opt.B, Quantum semiclass. Opt 4,Rl (2002)

[2]. $\quad$ R.Loudon and P.L.Night, J.Mod.Opt 34,709(1987)

[3]. M.J.Everitt,W.J.Munro,T.P.Spiller, Phys.Rev.A 85,022113(2012)

[4]. C.M.Caves and B.L.Schumaker, Phys.Rev.A 31,3068(1985)

[5]. J.H.Shapiro, Opt.Lett.5,351(1980)

[6]. H.P.Yuen,and,J.H.Shapiro, IEEE Trans.Inf.Theory 24,657(1978)

[7]. M.Hillery, Phys.Rev.A 61,022309(2009)

[8]. S.L.Braunstein,and,H.J.Kimble, Phys.Rev.A 61,042302(2000)

[9]. Gorbachev V N,Zhiliba A I and Trubiko A I, J.Opt.B, Quantum semiclass. Opt. 3,S25 (2001)

[10]. H Prakash and D K Misra Opt.Lett. 35,2212(2010)

[11]. S Rani et.al. Indian Journal of Physics 86,53(2012)

[12]. J Abadie et.al. Nature Physics 7,962-965(2011)

[13]. J Aasi et.al. Nature Photonics 7,613-619(2013)

[14]. R S Bondurant,P Kumar,J H Shapiro and M Maeda, Phys.Rev.A 30,343(1984)

[15]. W Becker, M O Scully and M S Zubairy, Phys.Rev.Lett.48, 475 (1982)

[16]. H Shi and M Bhattacharya, Phys.Rev.A 87, 043829(2013)

[17]. K Mukherjee, P C Jana, Journal of optics, 016, 0339 (2016)

[18]. T J Kippenberg and K J Vahala, Science 321,1172(2008)

[19]. F Marquardt and S M Grivin, Physics2,40(2009)

[20]. J Q Lio and F Nori, arxiv :1304.6612, quant-ph (2013)

[21]. H K Li, Y C Liu, X Yi,C L Zou, X X Ren and Y F Xiao, Phys.Rev.A 85,053832(2012)

[22]. X Hu,F Nori, Physica B 263-264(1999)16-29

[23]. Zhi-ming,Xu L,Chai J-L and Li F-L, Phys.Lett.A 150,27(1990)

[24]. M Hillery, Phys.Rev.A 40,3147(1989)

[25]. M Hillery Optics Communications 62,135-138(1987)

[26]. K Goda et.al. Nature Physics 4,472-476(2008)

[27]. A Sizmann et.al. Optics Communications 80,138-142(1990)

[28]. J Lal and R M P Jaiswal et.al. Indian Journal of Physics 72,637-642(1998)

[29]. A Kumar and P S Gupta J.Opt.B Quantum semiclass. Opt.8, 1053-1060(1996)

[30]. J Lal and R M P Jaiswal et.al. Indian Journal of Pure and Applied Physics 36,415-418(1998)

[31]. S Rani, J Lal and N Singh et.al. International Journal of Optics 629605,(2011)

[32]. J D Thompson, B M Zwickl, A M Jayich, F Marquardt, S M Grivin, and J G E Harris, Nature (London) 452, 72(2008)

[33]. H K Cheung and C K Law, Phys.Rev.A 84, 023812 (2011)

[34]. J C Sankey, C Yang, B M Zwickl, A M Jayich and J G E Harris, Nature Phys. 6,707(2010)

[35]. N E Flowers-Jacobs et.al. , Appl.Phys.Lett. 101, 221109(2012)

[36]. J Gieseler, B Deutsch, R Quidant,and L Novotny, Phys. Rev. Lett.109, 103603(2012)

[37]. S Bose, K Jacobs and P L Knight, Phys.Rev.A 56, 4175, (1997)

[38]. A Rai and G S Agarwal, Phys.Rev.A 78, 013831(2008)

[39]. P Rabl, Phys.Rev.Lett. 107, 063601(2011)

[40]. F Massel et.al. Nat.Photonics 3,987(2012) 